\section{A program for parsing mouse movements into component submovements}

\author{
NEFF WALKER, DAVID PHILBIN, \\ and AILEEN WORDEN \\ Georgia Institute of Technology, Atlanta, Georgia \\ and \\ JOHN B. SMELCER \\ American University, Washington, D.C.
}

\begin{abstract}
Most current movement control theories include the idea that movement toward a target can be broken into several submovements. The complexity of analyzing a movement into its constituent submovement structure and the additional complexity imposed by the problem of noise in the data and hand tremor seem to be daunting to researchers. This paper discusses a program that can ameliorate both of these problems and parse movements into their constituent submovements. It also contains a graphing feature that is useful as a visual tool for analyzing submovement structure. The programs are easily modifiable, so that researchers can specify their own parsing rules on the basis of different assumptions about movement control and use the parser for data from different experimental tasks.
\end{abstract}

During the last few years, the use of computer mice to collect movement control data has increased (see, e.g., Baccino \& Pynte, 1994; Walker, Meyer, \& Smelcer, 1993). This is partly due to recent articles about the underlying structure of the data stream that researchers gather with computer mice and software (e.g., Crosbie, 1990). In addition, there are custom-designed programs available that can be used in conjunction with the commercial software to extend the type and quality of data that can be obtained using a computer mouse (e.g., Baccino \& Kennedy, 1995; Segalowitz \& Graves, 1990).

Although these programs and extensions make the computer mouse a good research tool, they do not provide a means for parsing movement data into a component submovement structure. This is a major drawback because the distinctions between various movement control theories are expressed in terms of submovement structure (see, e.g., Crossman \& Goodeve, 1983; Jagacinski, Repperger, Moran, Ward, \& Glass, 1980; Meyer, Abrams, Kornblum, Wright, \& Smith, 1988). Parsing programs must perform three major tasks in order to yield consistent data on submovement structure. First, the program must smooth and filter the data stream to eliminate noise. Second, rules that define submovements must be applied to this stream, producing a consistent and rich database on this structure. Finally, the parsing

B.S. is at the Kogod School of Management, American University. Correspondence should be addressed to N. Walker, School of Psychology, Georgia Institute of Technology, Atlanta, GA 30332-0170. program should be flexible enough to handle different data sources (e.g., mouse data collected with different sampling rates) and to allow the researcher to easily identify and test specific rules for parsing movements into a constituent submovement structure.

In this paper, we describe a set of Pascal programs that smooth, differentiate, and parse movement data into a rich profile of underlying submovement structures. It is important to review the model of submovement structure because it was used in the development of these programs. Then we describe six major components of the programs.

\section{Model of the Submovement Structure of Mouse Movement}

Historically, most theories of movement control have assumed that aimed movements consist of two phases (see, e.g., Woodworth, 1899). The first phase of movement is initial impulse and is characterized by a programmed ballistic movement. The first phase of movement is followed by a current control phase in which feedback is used to guide corrective movement toward a target. This secondary phase of movement can be defined by changes in velocity and acceleration. In the last 30 years, this twophase model has been expanded to include the possibility of many corrective submovements in the current control phase (see, e.g., Crossman \& Goodeve, 1983; Jagacinski et al., 1980). In addition, a blurring of the distinction between the initial ballistic movements and subsequent corrective movements has occurred (Meyer et al., 1988). Our set of programs was built on the basis of assumptions of submovement structure proposed by Jagacinski et al. (1980) and Meyer et al. (1988; Meyer, Smith, Kornblum, Abrams, \& Wright, 1990).

The idea behind this theoretical approach is that overall movements from one location to another consist of a series of discrete submovements. The number of submovements in the overall movement may vary, but each submovement can be determined by looking at values and changes of velocity, acceleration, distance of movements, and duration of movements. These variables are used to specify the muscle pulses that define secondary movements while eliminating muscle impulses due to tremor or other noise. The specific numerical rules we have used to define submovements are discussed later in the paper. Our set of programs uses these rules to parse movements into submovement structures to fit most theoretical explanations of movement control (e.g., Jagacinski et al., 1980; Keele, 1968; Meyer et al., 1988).

Reading the input files. The first program (dfiltner) is set up to accept data from any input device that can give $x$ and $y$ coordinates and a time resolution stamp. The parsing programs also accept an attached header line for each movement trial that pertains to experimental conditions. For example, the subject number, trial, and distance and width of the target are usually included in the 
Start of Trial

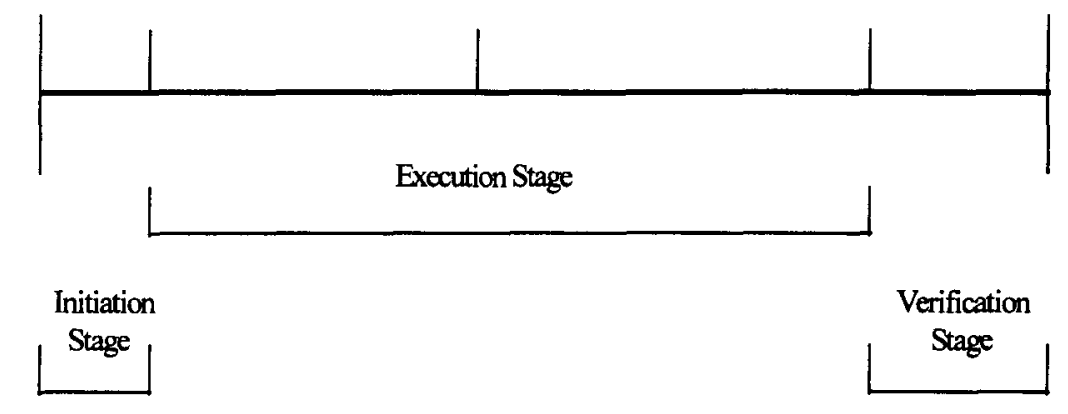

End of Trial

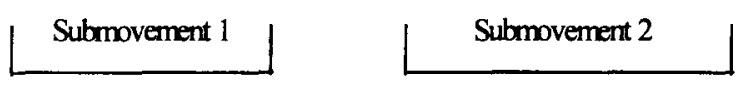

Pause

Time

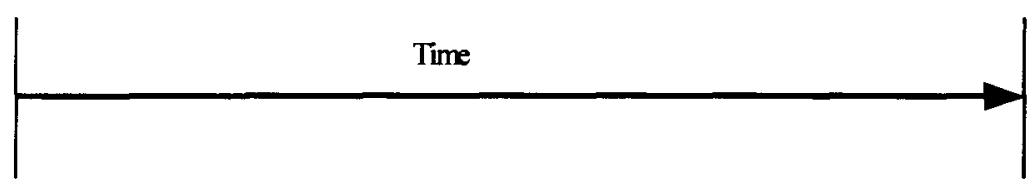

Figure 1. Component structure of cursor movements.

header. The program skips this header and begins on the first line of position output. Each line of position output provides an $x$ and $y$ coordinate for the cursor and a time stamp. The program reads the data until it reaches a line containing an end of file code (e.g., 9999990 ). Both the header lines and the end of file codes are ignored for the purpose of smoothing and differentiating the data files. The header file is added back to the output for the parsing program (see Read_Data).

Smoothing the data streams. Noise in the data is a common problem with studies that analyze mouse movements. Noise comes from two primary sources: (1) tremor and (2) data recording. Specifically, mouse software does not record a continuous data stream. Instead, it samples the mouse position at fixed intervals. The sampling rate commonly ranges between 5 and $20 \mathrm{msec}$ for a bus mouse. This type of data stream produces discrete position information. For example, a cursor being moved at a constant 25 pixels per second, for $1 \mathrm{sec}$, measured at $20-\mathrm{msec}$ intervals, would produce 50 samples. Alternating samples in this case would reflect an absence of change in position, which indicates intermittent zero velocities. The data in the above example indicate 25 absence-of-velocity samples that could be misinterpreted as submovements. The result is noise in the data that can make submovement parsing problematic. Our programs (dfiltner and dfltnrd) smooth the data stream and then filter out the noise before parsing the data.
The dfiltner program computes tangential distances for each movement. Tangential distance is computed as the Euclidean distance between adjacent movements. Therefore, tangential distance is always positive. The data are then smoothed with the NER digital filter of Kaiser and Reed (1977). The smoothing filter has a $0-$ to $29-\mathrm{Hz}$ passband, a 29- to $31-\mathrm{Hz}$ skirt, and a stopband from $31 \mathrm{~Hz}$ upward. This filter reduces the effects of hand tremor and jittery movements due to mouse friction.

Differentiating the data. The smoothed data are then differentiated once with a $30-\mathrm{Hz}$ low-pass filter (dfltnrd) to give velocities and then again to give accelerations for continuous points in time. The product is a continuous record of position, velocity, and acceleration that defines submovement structures for the parser. For details of how and why this approach is used, see Kaiser and Reed (1977).

Parsing the data. The data are parsed by our program after they are smoothed and differentiated. The easiest way to understand the parser is to refer to Figure 1. As the figure shows, we assume that movements can be broken into a hierarchy of components. The first level of the hierarchy is the total movement. Our parser assumes that the beginning and ending of total movement is marked by either a machine signal or a user-initiated action. The total movement is broken into an initiation stage (GetReactionTime), an execution stage, and a verification stage (GetButtonReleaseTime). Execution time is simply total 


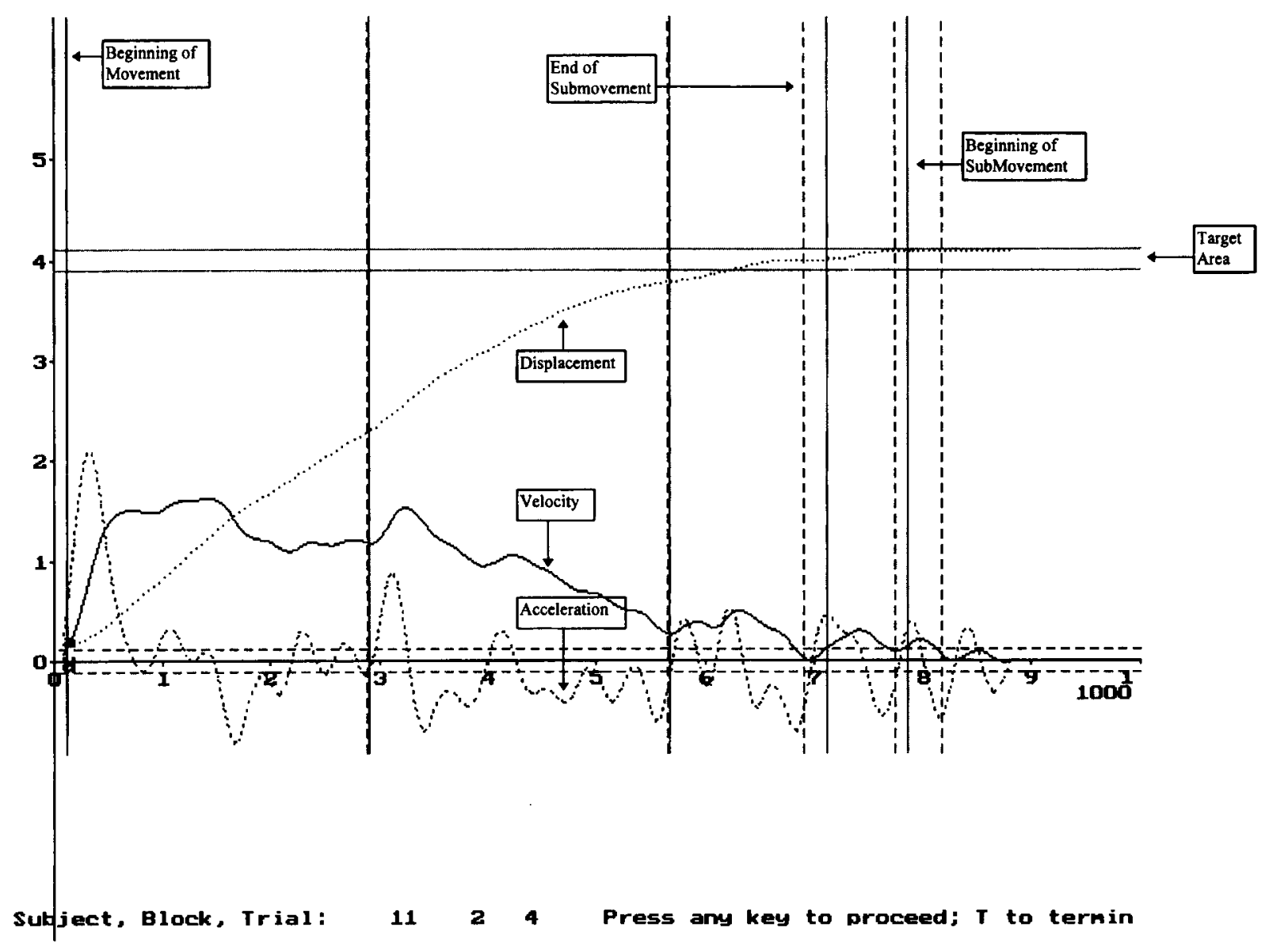

Figure 2. Example graph output of the parser.

movement time minus times for the initiation and verification stages of movement. The second level of the hierarchy represents the submovements and pauses that constitute the execution stage of movement.

The parser locates and records the initiation, execution, and verification stages. It also identifies the submovements (Parse) and pauses of the execution stage (Parse_Deadtime). Initiation time is the time between when the trial begins and the start of the movement. The execution stage begins with the first submovement (GetFirstSM). The beginning of a submovement is recorded when $20 \mathrm{msec}$ of motion with a velocity greater than 75 pixels/sec is detected (see GetFirstSM and DeadTimeEnd). The end of each submovement is determined by an algorithm that uses relative velocities and accelerations (SubMovementEnd). Indications of submovement termination include velocities that cross zero and changes in acceleration vectors. For the latter indication of submovement termination, relative minimum velocity must be at least $20 \%$ less than the relative maximum velocity of that submovement. These conditions ensure that small fluctuations in velocity are not considered submovements. These values were selected on the basis of previ- ous theoretical work (Jagacinski et al., 1980; Meyer et al., 1988), but they can all be altered if the researcher wants to use different rules and cutoff values to define the beginning or end of a submovement.

The end of a submovement marks the start of a pause. Pauses are the times when the mouse is motionless between submovements. Pauses end when additional submovements are detected or the end of a trial is indicated by the release of the mouse button. The pause between the end of the last submovement and the release of the mouse button is considered verification time (GetButtonReleaseTime).

The program also allows two different sets of rules for defining submovements. The reason for allowing multiple ways to define submovements is that some movement tasks require very fine control at the end of movement (e.g., placing the cursor on a very small target). These small submovements may not reach the minimum velocity and distance criteria. The parsing program's Submovement_Type specifies the submovement type. The two submovement types are Ballistic and Smallslow. The distinction between types of submovements is made by having different minimum distance, velocity, and du- 
ration parameters. This distinction in movement type was implemented because in movement tasks that require precise endpoint location of the movement, many small and corrective submovements may be required. The features (e.g., velocity, duration, and distance) that define these small corrective submovements can be different from those that define other submovements. In the current version of the parsing program, all of this code has been commented out because most tasks do not require this distinction. However, we did not delete this code because it may be appropriate for some types of data analysis.

All of the parameters that are built into the program to define the beginning and end of submovements are usually set as variables and can be changed. The current values were set because we found them to produce the best results with our experimental data (e.g., Walker et al., 1993; Walker, Philbin, \& Fisk, 1997). Changes may be needed if the mouse samples at a different rate or the pixel size is different, but we have found the program to be usable for data collected using different mouse drivers (two versions of Microsoft up to Version 9.01 and one version of Logitech drivers) and different sampling rates. In our previous work, we have primarily used a Microsoft bus allowing us to sample at $200 \mathrm{~Hz}$ (Walker et al., 1993), but we have also tested the parser program on data collected at lower sampling rates. The programs can be adjusted to allow different sampling rates to be used (as with the data from a serial port mouse).

Graphing the structure of movement. The graphing program was implemented as a way to display the rules used to define submovements. The graphing was designed to aid in the evaluation of the rules chosen to define submovements, which is important because rules may not be the same for all movement tasks.

In Figure 2, the $x$-axis represents time and is labeled in hundreds of milliseconds. The $y$-axis represents values of displacement, velocity, and acceleration. The scales on both axes are automatically adjusted to fit in available screen space with the available graphics mode (e.g., VGA, SVGA).

The primary purpose of the graph function is to allow the user to see how the rules for defining submovements map onto the actual data for any trial. The graphing plots displacement, velocity, and acceleration across time. Displacement is represented by dotted lines (white), velocity by a solid line (white), and acceleration by a dashed line (blue). The graph function also marks the beginning and end of submovements with vertical lines. The beginning of a submovement is represented by a solid vertical line (green), and the end of a submovement is represented by a dotted vertical line (red).

The location on the $y$-axis represents the distance the cursor must travel. Distance between the two lines represents the size of the target. The graph also includes a pair of horizontal solid lines (white) that represent the target area at the appropriate location on the $y$-axis. This infor- mation allows the user to quickly identify movement errors, overshoots, and undershoots. For the graphing function to work, one must ensure that the location of information used by the graphing function (target location and distance of movement) can be extracted from the header file. The program looks for variables in specific locations in the header, and the program must be past the correct locations. This information is contained in the Parse_Header procedure.

The idea behind the graphing function is that the display allows researchers to see how their parsing rules are applied to the data. On the basis of this visual inspection, the researcher can decide to use the selected rules or alter the rules used in the parser. For example, in Figure 2, one can see that the end of the first submovement and the beginning of the second submovement occur at an inflection point for both velocity and acceleration. A researcher can look at the output and determine whether the rules used to define the submovement structure are appropriate. One of the parameters passed to the parsing program determines whether the graphing function is enabled during parsing, which is useful because the graphing function is not necessary once the parsing rules have been confirmed.

Saving the parser output. The program creates an ASCII data file (Save_Data) that contains header information and data produced by the parser. The data produced by the parser are organized to reflect the hierarchy of movement. The parser stores total movement time, maximum and average velocity, maximum and average acceleration, final cursor position, and number of submovements. The program also stores the time for the initiation and verification stages of movement.

Data for submovements are organized to reflect multiple submovements and pauses between submovements. The program records data for up to 10 submovements and pauses per trial. Time, distance moved, average velocity, maximum velocity, average acceleration, and maximum acceleration are recorded for each submovement. When fewer than 10 submovements are made, missing data codes are stored (we use $9 \mathrm{~s}$ to fill the variable field).

In addition, the program contains commented code that can add variables to the output. Additional variables include time to peak acceleration, deviation from a straight line between starting location and movement target, and distance from the target at the end of each submovement. These variables can be added to the program output by removing comment markers from the source code.

\section{Program Availability}

The source code for the set of programs described in this paper is available via FTP (ftp.cc.gatech.edu/pub/ software/mouse_parser). The information available via FTP includes the programs required to produce the output we have described, a readme file, and a sample batch file to automate the process of feeding data from one program (e.g., dfiltner) to the next program (dfltnrd). 


\section{Summary and Conclusions}

This paper was written to introduce a tool that can be used effectively to analyze and present movement control data. We believe that this set of programs is a useful and necessary research tool for those doing work on movement control. In the introduction of the paper, we specified three criteria that we felt a good parsing program should meet. We believe that the programs we have created meet these criteria. First, the programs eliminate the problem created by a discrete, intermittent data stream and also filter out the noise caused by hand tremors. Second, the algorithms that are currently installed in the program allow submovements to be identified efficiently, while the graphing aspect of the program displays the submovements visually. It is not necessary to subscribe to a particular model of submovement structure for this program to be useful. The source code is easily adjusted to fit various submovement algorithms, which can be designed or altered by the individual researcher. Third, the programs produce a wide range of dependent measures. Few researchers will need or want all of this information stored in their data files, but because researchers will differ in their specific data needs, a broad range of variables is necessary; researchers can select the variables to be saved by using comment markers in the code.

Finally, we think that the ability to determine the submovement structure of cursor movement has application beyond the area of human-computer interaction and would be useful to many types of researchers in the fields of psychology, human factors, and engineering. For example, work in the area of aging often investigates age-related differences in movement control, but few researchers in that area have analyzed submovement structure. The program could also be used to analyze the cursor movement that is required in a computer simulation, allowing the research to separate and analyze the role that these movements play in overall performance. The program would also allow researchers in more basic areas of psychology (e.g., memory search tasks) to investigate the specific motor components of their task more fully.

\section{REFERENCES}

Baccino, T., \& Kennedy, A. (1995). MICELAB: Spatial processing of mouse movement in Turbo Pascal. Behavior Research Methods, Instruments, \& Computers, 27, 76-82.

Baccino, T., \& PYNTE, J. (1994). Spatial coding and discourse models during text reading, Language \& Cognitive Processes, 9, 143-155.

Crosbie, J. (1990). The Microsoft mouse as a multipurpose response device for the IBM PC/XT/AT. Behavior Research Methods, Instruments, \& Computers, 22, 305-316.

Crossman, E. R. F. W., \& Goodeve, P. J. (1983). Feedback control of hand-movement and Fitts' law. Quarterly Journal of Experimental Psychology, 35A, 251-278.

JaGacinski, R. J., Repperger, D. W., Moran, M. S., Ward, S. L., \& GLASS, B. (1980). Fitts' law and the microstructure of rapid discrete movements. Journal of Experimental Psychology: Human Perception \& Performance, 6, 309-320.

KAISER, J. F., \& REED, W. A. (1977). Data smoothing using low-pass digital filters. Review of Scientific Instruments, 48, 1447-1457.

KEELE, S. W. (1968). Movement control in skilled motor performance. Psychological Bulletin, 70, 387-403.

Meyer, D. E., Abrams, R. A., Kornblum, S., Wright, C. E., \& Smith, J. E. K. (1988). Optimality in human motor performance: Ideal control of rapid aimed movements. Psychological Review, 95, 340-370.

Meyer, D. E., Smith, J. E. K., Kornblum, S., Abrams, R. A., \& WRIGHT, C. E. (1990). Speed-accuracy tradeoffs in rapid aimed movements: Toward a theory of rapid voluntary action. In $\mathrm{M}$. Jeannerod (Ed.), Attention and performance XIV (pp. 173-226). Hillsdale, NJ: Erlbaum.

Segalowitz, S. J., \& Graves, R. E. (1990). Suitability of the IBM XT, $\mathrm{AT}$, and PS $/ 2$ keyboard, mouse, and game port as response devices in reaction time paradigms. Behavior Research Methods, Instruments, \& Computers, 22, 283-289.

WALKer, N., MeYer, D. E., \& SMelCer, J. B. (1993). Spatial and temporal characteristics of rapid cursor-positioning with electromechanical mice in human-computer interaction. Human Factors, 35, 431-458.

WAlKer, N., Philbin, D. A., \& Fisk., A. D. (1997). Age-related differences in movement control: Adjusting submovement structure to optimize performance. Journal of Gerontology: Psychological Sciences, 52B, 40-52.

WOODWORTH, R. S. (1899). The accuracy of voluntary movement. Psychological Review, 3 (2, Whole No. 13).

(Manuscript received April 12, 1996; revision accepted for publication October 10, 1996.) 